\title{
Communication environnementale et réputation de l'organisation
}

Dans cet article, les auteurs avancent l'idée que les informations publiées par l'organisation sur son comportement environnemental constituent des signaux qui auront des impacts différenciés sur la réputation organisationnelle en fonction de leur nature, leur visibilité et leur cohérence. Les résultats indiquent que la conformité, la coprésence de signaux conformes et non conformes, et la visibilité du signal rejaillissent favorablement sur la réputation. Enfin, l'impact des signaux de conformité peu visibles dépend de la réputation antérieure de l'organisation, et ce à l'avantage des organisations bénéficiant d'une réputation plus élevée que la moyenne. Ces résultats permettent ainsi de contribuer à la fois à la littérature sur la communication environnementale et à celle sur le management de la réputation. 
$\mathrm{L}$ a réputation d'une organisation désigne la manière dont l'organisation est perçue et évaluée par ses parties prenantes quant à sa capacité à générer de la valeur comparativement à ses pairs et concurrents (Deephouse, 2000; Fombrun, 1996; Fombrun et Shanley, 1990; Rindova et al., 2005). Si les conséquences positives d'une bonne réputation semblent bien comprises et largement confirmées empiriquement dans la littérature (Rindova et al., 2005 ; Roberts et Dowling, 2002), nous pouvons cependant remarquer que la question de la construction de la réputation a reçu une attention plus marginale. Nous nous intéressons donc dans ce papier aux antécédents de la réputation organisationnelle et cherchons à prolonger les travaux pionniers en la matière (Fombrun et Shanley, 1990; Rindova et al., 2005). Plus précisément, nous nous intéressons à la question de recherche suivante: dans quelle mesure la communication environnementale d'une organisation peut-elle avoir un impact sur sa réputation? Par communication environnementale, nous entendons toute information relative à l'empreinte écologique de l'organisation sur l'environnement naturel dans lequel elle évolue (Wilmshurst et Frost, 2000). Quant à la réputation, nous la définissons comme l'agrégation dans la durée des diverses perceptions d'une organisation par ses parties prenantes (Fombrun et Shanley, 1990).

Il nous paraît intéressant de nous pencher sur la communication environnementale des organisations car nous pouvons observer depuis les années 1990 une montée en puissance des attentes en matière de responsabilité environnementale (Bansal, 2005) donnant lieu à une demande de conformité elle-même intrinsèquement liée à la production de textes par l'organisation. Or ces textes s'avèrent fort utiles comme base d'études empiriques (Deephouse et Carter, 2005). Il est par ailleurs également important d'un point de vue théorique de s'intéresser aux questions de responsabilité sociétale (au sens large) des organisations car elles représentent une composante de plus en plus cruciale dans la formulation de leur stratégie (Crane, 2000).

En effet, la responsabilité environnementale constitue un investissement stratégique pour l'organisation, qui espère retirer des bénéfices non seulement de son implication dans des activités de responsabilité sociale (Baron, 2001; Martinet et Reynaud, 2001) mais également de sa communication sur cette implication. Si la littérature sur le sujet a largement exploré l'existence de liens entre responsabilité sociétale et performance financière des organisations (Gardberg et Fombrun, 2006; McGuire et al., 1988; Waddock et Graves, 1997), elle s'est beaucoup moins intéressée sur un plan empirique à la question d'un lien entre responsabilité sociétale et réputation organisationnelle, alors même que nombre de travaux suggèrent qu'une organisation peut améliorer sa réputation en adoptant des pratiques responsables (Fombrun, 1996; Gardberg et Fombrun, 2006; Martinet et Reynaud, 2001). En nous intéressant au lien existant entre la communication environnementale d'une organisation et sa réputation, nous cherchons également à combler un autre manque de la littérature en théorie des organisations. La gestion de la réputation repose largement sur la communication organisationnelle (Fombrun, 1996) et l'utilisation de symboles évocateurs (Westphal et Zajac, 1998), pourtant peu de recherches se sont intéressées préci- 
sément à la manière dont les actions symboliques des organisations (communication orale et écrite par exemple) affectent directement et substantiellement certaines variables organisationnelles stratégiques (Suddaby et Greenwood, 2005). Nous cherchons donc dans ce papier à combler ces manques dans la littérature grâce à une étude empirique de l'impact de la communication environnementale d'une organisation sur sa réputation.

Nous nous appuyons dans ce papier sur une perspective double, à la fois économique via la théorie des signaux et sociologique via le néo-institutionnalisme pour conceptualiser la communication environnementale comme un mécanisme institutionnel permettant à l'organisation d'émettre des signaux de conformité à destination de ses parties prenantes. Par conséquent, nous nous intéressons au lien de causalité entre la communication environnementale et la construction de la réputation organisationnelle.

\section{I - CADRE THÉORIQUE}

La réputation joue un rôle crucial dans la structuration des marchés et génère des bénéfices économiques et institutionnels pour les organisations. En fonction de leur réputation, les organisations vont élargir ou restreindre leur champ d'action stratégique. Ainsi, une alliance est plus souvent contractée entre organisations de même niveau de réputation qu'entre organisations de rangs différents (Benjamin et Podolny, 1999), les coûts financiers supportés par une organisation diffèrent selon la réputation de celle-ci (Fombun, 1996) et les risques financiers sont tempérés par une bonne réputation (Bansal et Clelland, 2004).
La recherche sur les antécédents de la réputation identifie un grand nombre de facteurs économiques et sociaux (Durand et al., 2007; Pollock et Rindova, 2003) contribuant à la construction de cet actif stratégique. Dans une méta-analyse de 60 études, Rindova et al. (2005) identifient deux traditions de recherche qui emploient le construit de réputation organisationnelle: les littératures économiques et institutionnelles. La première définit la réputation comme l'évaluation d'un attribut (e.g. la qualité d'un produit) permettant de réduire l'incertitude. La seconde associe la réputation à une perception et une reconnaissance collective de l'organisation. Dans ce papier, nous nous appuyons sur ces deux traditions qui ne sont pas mutuellement exclusives et se complètent même efficacement.

D'un point de vue économique, la réputation représente le résultat d'un processus concurrentiel au cours duquel une organisation va signaler ses caractéristiques clés à ses diverses audiences afin de maximiser sa valeur économique et sociale (Fombrun et Shanley, 1990). Ainsi la théorie des signaux développe-t-elle l'idée qu'une organisation peut réduire des problèmes d'asymétrie d'information en envoyant et interprétant des signaux (Spence, 1974). Les asymétries d'information surviennent lorsque l'une des parties possède des informations dont l'autre ne dispose pas, ce qui conduit les différents acteurs à chercher/envoyer des signaux permettant de fournir des informations sur ce qui ne peut être observé. On peut donc définir un signal comme un attribut ou une activité qui prouve que l'une des parties dispose d'informations privées et difficilement vérifiables et qui peut aider l'autre partie à réduire son incertitude quant à la qualité intrinsèque mais non observable 
de l'émetteur (Spence, 1974). Pour être crédible et interprété comme indicateur de qualité, un signal doit être coûteux à émettre et doit requérir un fort engagement de la part de l'émetteur (Spence, 1974). La théorie des signaux suggère également que les parties informées émettent activement des signaux tandis que les audiences non informées reçoivent et interprètent passivement ces signaux. Mais dans des conditions de grande incertitude, les audiences peuvent se mettre en quête active de filtres des attributs organisationnels tels que le capital social, des affiliations particulières, ou la réputation - leur permettant d'opérer un tri entre les différentes organisations émettrices (Weiss, 1995).

Les facteurs économiques ne sont pas seuls à influencer la manière dont les audiences évaluent les organisations. Ainsi C.J. Fombrun et M. Shanley (1990) affirment-ils que des facteurs sociaux ont un rôle crucial à jouer dans la construction de la réputation. D'un point de vue institutionnaliste, les organisations cherchent à démontrer la congruence entre leurs pratiques et les valeurs professées par leur environnement institutionnel (Oliver, 1999) et par conséquent adoptent des pratiques conformes aux normes sociales (Meyer et Rowan, 1977). Dès lors, la littérature institutionnaliste suggère que la communication d'une organisation constitue une réaction aux pressions exercées sur elle par son environnement institutionnel, en ce sens où l'organisation va utiliser sa communication pour influencer les perceptions de ses audiences afin d'apparaître socialement désirable et d'être favorablement évaluée (Oliver, 1991).

Dans le contexte de la performance environnementale, la communication devient cruciale pour deux raisons. D'une part, cette performance repose dans une large mesure sur l'habileté d'une organisation à gérer ses interactions avec son environnement institutionnel et donc à établir un dialogue avec ses parties prenantes. D'autre part, il est souvent difficile pour les parties prenantes de l'organisation d'évaluer précisément sa performance en matière de responsabilité sociale et particulièrement environnementale (Baron, 2001), et celles-ci dépendent donc largement des informations publiées par l'organisation pour se former une opinion quant à la qualité intrinsèque de ses actions.

\section{II - HYPOTHÈSES}

\section{Nature des signaux et réputation}

Une bonne réputation permettant de bénéficier d'avantages directs et indirects, les organisations vont s'efforcer d'améliorer leur capital de réputation en effectuant des choix symboliques visibles, tels que le choix d'un nouveau nom (Glynn et Abzug, 2002), ou des investissements importants dans des activités assurant la préservation de l'environnement (Bansal et Clelland, 2004; King et Lenox, 2005). Des auteurs issus de différentes traditions de recherche convergent pour dire que les organisations qui cherchent de manière proactive à aligner leurs activités avec leur environnement institutionnel au travers de l'évocation de symboles et d'actions appropriés, parviennent à améliorer leur réputation (Bansal, 2005; Durand, Rao, et Monin, 2007; Rindova et al., 2005).

Ainsi les organisations vont-elles utiliser leur communication environnementale (Wilmshurt et Frost, 2000) pour mettre en exergue la congruence entre leurs valeurs et 
actions et celles attendues par la société (Suchman, 1995), afin d'améliorer leur réputation (Deephouse et Carter, 2005) et recueillir les bénéfices afférents. Étant donnée l'existence de fortes pressions isomorphiques autour du respect des normes et des valeurs, les organisations qui s'engagent dans des comportements non conformes aux attentes de la société sont susceptibles de se voir sanctionnées par leur environnement institutionnel (DiMaggio et Powell, 1983). Cela incite donc les organisations à envoyer des signaux de conformité à leurs audiences afin de construire, maintenir ou renforcer leur réputation (Suchman, 1995). La communication environnementale peut être interprétée comme un signal indiquant que le comportement de l'organisation est socialement acceptable et approprié, ce qui devrait conduire à améliorer la réputation de l'organisation. Communiquer sur des événements de non-conformité vis-à-vis des attentes de la société, devrait au contraire impacter négativement la réputation de l'organisation. Nous suggérons donc les hypothèses suivantes:

Hypothèse 1a. Les organisations qui envoient des signaux de conformité améliorent leur réputation.

Hypothèses 1b. Les organisations qui envoient des signaux de non-conformité détériorent leur réputation.

\section{Visibilité des signaux et réputation}

Dans un contexte de maturité institutionnelle, où chaque organisation est consciente des attentes de la société en matière de responsabilité environnementale des organisations, la nature des signaux (conforme ou non conforme), bien qu'essentielle, n'est pas suffisante pour que le signal puisse être interprété comme un indicateur de qualité. Les attentes environnementales intervenant sur un plan normatif, chaque organisation est incitée en théorie à apparaître socialement désirable sur ce plan. La manière dont les informations vont être publiées et présentées au public devient donc stratégique. En nous fondant sur la théorie des signaux concurrentiels développée par O. Heil et T.S. Robertson (1991), nous suggérons que l'interprétation des signaux ne va pas seulement dépendre de la nature de l'information véhiculée, mais également des caractéristiques du signal envoyé, et spécifiquement de sa visibilité. Un signal visible est un signal qui peut être perçu et interprété rapidement avec une faible marge d'erreur. Le récepteur est donc capable d'évaluer avec plus d'acuité et de certitude les véritables intentions de l'organisation émettrice. Cela nous conduit donc à distinguer entre signaux de conformité visibles et peu visibles. Nous considérons comme signaux visibles des informations (événements, données, chiffres) qui bénéficient d'une bonne proéminence au sein de la communication générale émise par l'organisation, en ce sens où cette proéminence les rend aisément repérables et interprétables par le récepteur. Au contraire, nous considérons comme signaux peu visibles, des informations qui seraient peu saillantes au sein de la communication générale de l'organisation, qui ne représenteraient qu'une petite fraction des informations émises, et qui seraient donc difficiles à repérer et identifier par le récepteur. Ces deux types de signaux sont utilisés par les organisations pour influencer les perceptions de leurs audiences, mais nous suggérons qu'ils auront des impacts différenciés. D'où l'hypothèse suivante : 
Hypothèse 2. Les signaux de conformité visibles ont un impact positif plus grand que les signaux de conformité peu visibles sur la réputation de l'organisation.

Nous pouvons par ailleurs noter le peu d'intérêt porté dans la littérature à la question de la cohérence des signaux émis et de l'impact de cette cohérence sur des variables stratégiques telles que la réputation (e.g. Durand et al., 2007). La plupart des travaux semblent en effet considérer que les organisations sont peu susceptibles d'envoyer simultanément des signaux divergents, à savoir dans notre cas, des signaux de conformité et de non-conformité aux attentes en matière de performance environnementale. Une extension logique de la littérature consiste donc en l'investigation de l'impact de la présence simultanée de signaux conformes et non conformes sur la réputation de l'organisation. Nous suggérons donc ici que la présence d'un signal visible de conformité devrait positivement modérer l'impact négatif d'un signal de conformité sur la réputation de l'organisation. En effet, la simple présence d'information positive peut conduire les récepteurs à ajuster leurs évaluations. Par ailleurs, la visibilité du signal de conformité est susceptible d'exercer une influence positive modératrice dans la mesure où elle accroît la crédibilité de l'organisation et peut contribuer ainsi à normaliser les informations divergeant des attentes institutionnelles (Suchman, 1995). Enfin, la présence d'informations positives et visibles devrait contribuer à circonscrire les informations de non-conformité à une partie de l'organisation en isolant cette partie par rapport aux efforts par ailleurs menés en matière de responsabilité environnemen- tale (Elsbach et Sutton, 1992). Nous émettons donc 1'hypothèse suivante:

Hypothèse 3. La présence d'un signal visible de conformité va modérer positivement la relation négative entre la présence d'un signal de non-conformité et la réputation de l'organisation.

\section{Crédibilité de l'émetteur et réputation}

Jusqu'à présent, notre discussion s'est exclusivement focalisée sur les caractéristiques intrinsèques des signaux, en suggérant que leur nature et leur visibilité sont susceptibles d'influencer la manière dont ils seront interprétés. Cependant, dans des contextes d'asymétrie informationnelle, les économistes distinguent généralement entre deux types d'équilibres: séparateur et non séparateur (Milgrom et Roberts, 1982). Dans un équilibre séparateur, les émetteurs de différents types (de différentes qualités) vont choisir d'émettre des signaux différents, permettant ainsi aux récepteurs de distinguer les émetteurs en inférant la qualité sous-jacente de ceux-ci. Cependant, il peut arriver que des émetteurs de différents types choisissent d'émettre le même signal, ce qui ne permet plus aux récepteurs de distinguer les émetteurs selon leur type. Dans une telle situation d'équilibre non séparateur, les récepteurs ne peuvent plus considérer les signaux comme des substituts de la qualité car ils ne transmettent pas d'information crédible. Pour distinguer entre signaux crédibles ou non et donc évaluer la qualité de l'organisation émettrice, les récepteurs doivent donc se reposer sur l'utilisation de filtres.

Nous considérons que la communication environnementale des organisations pourrait être concernée par ce problème d'équilibre non séparateur, étant donné le contexte institutionnel fortement normatif qui incite 
les organisations à publier des informations abondantes et relativement standardisées. Nous suggérons ici que les signaux de conformité visibles ne devraient pas être touchés par ce problème car leur visibilité les rend facilement interprétables et vérifiables par les diverses audiences de l'organisation (Heil et Robertson, 1991) et par conséquent crédibles. L'envoi de signaux de conformité visibles qui ne seraient pas en adéquation avec la réalité serait en effet facilement détectable et puni. Nous suggérons au contraire, que la crédibilité des signaux de conformité peu visibles est plus questionnable, dans la mesure où ces signaux sont moins facilement interprétables, ne requièrent pas le même degré d'investissement de la part de l'organisation émettrice. Leur véracité est ainsi plus difficile à établir (Heil et Robertson, 1991; Spence, 1974). Le contenu de ces signaux peut donc être considéré comme nul en termes de transmission d'information quant à la réalité sous-jacente. Nous suggérons donc que les différentes parties prenantes vont tendre à utiliser des filtres pour évaluer la crédibilité de ces signaux environnements peu visibles.

Elsbach et Sutton (1992) et Elsbach (1994) montrent que les narrations subjectives des organisations à propos d'éléments jugés illégitimes par l'environnement institutionnel améliorent la manière dont ces éléments vont être perçus et acceptés par les diverses audiences de l'organisation, à partir du moment où l'organisation en question a atteint un certain niveau de reconnaissance publique. De la même manière, nous émettons l'hypothèse qu'il existe un seuil de réputation, à partir duquel les signaux peu visibles de conformité en matière de performance environnementale vont être jugés cré- dibles par les récepteurs. En effet, les organisations souffrant d'une réputation inférieure à celles des autres, font face à des pressions isomorphiques plus fortes (Phillips et Zuckerman, 2001) sont donc plus enclines à envoyer des signaux de conformité qui ne seraient pas en parfaite adéquation avec la réalité. Cependant, la réputation d'une organisation dépend de la consistance de ses actions dans la durée (Weigelt et Camerer, 1988) et sera donc négativement impactée si les signaux envoyés ne correspondent pas à la réalité (Kreps et Wilson, 1982; Milgrom et Roberts, 1982). Les organisations bénéficiant d'une réputation relativement élevée sont donc incitées à envoyer exclusivement des signaux en parfaite adéquation avec leur performance réelle. Il s'ensuit donc que les signaux de conformité peu visibles devraient avoir un impact positif sur la réputation de l'organisation si celle-ci bénéficie déjà d'une bonne réputation mais ne devraient pas avoir d'impact pour les organisations de réputation moins élevée, ce que nous formulons dans les hypothèses suivantes:

Hypothèse 4a. Pour les organisations ayant une réputation élevée, les signaux de conformité peu visibles ont un impact positif sur la réputation.

Hypothèse 4b. Pour les organisations ayant une réputation basse, les signaux de conformité peu visibles n'ont pas d'impact sur la réputation.

\section{III - MÉTHODOLOGIE}

\section{1. Échantillon}

Nous avons testé ces hypothèses sur la communication environnementale d'un échantillon de 90 entreprises américaines de 2001 à 2004 au moment où la tenue du Sommet 
de Johannesbourg en 2002 a suscité un intérêt marqué pour les questions relatives à la responsabilité environnementale des entreprises. La communication des organisations sur leur comportement environnemental devrait donc revêtir un aspect très stratégique durant cette période d'observation.

L'échantillon est issu de la liste publiée annuellement par le magazine Fortune sous le nom d'“America's Most Admired Companies". Cette liste présente le double avantage d'offrir un score de réputation pour chaque organisation et de permettre la constitution d'un échantillon transindustrie. La liste couvre en effet 11 secteurs : industrie financière, produits de consommation, services contractuels, distribution, informatique et communication, ressources naturelles, énergie, équipements de précision, équipements pour la maison, médias et divertissements, et transports. Nous avons aléatoirement sélectionné un quota d'organisations au sein de chaque secteur afin d'obtenir un échantillon de 100 entreprises. Cet échantillon a finalement été ramené à 90 entreprises, en raison de données manquantes, après avoir vérifié que l'exclusion de ces 10 entreprises n'altérait pas la structure de l'échantillon.

Nous avons collecté des données supplémentaires via différentes sources, la principale étant constituée par les rapports annuels des 90 organisations. Outre ces rapports annuels, nous avons également recueilli les rapports environnementaux/ développement durable émis par ces organisations via leurs sites internet et la base de données Thomson Research. Enfin, nous avons consulté COMPUSTAT afin d'obtenir des données de performance financière. Nous nous sommes particulièrement intéressés aux rapports annuels des organisations car lorsqu'il s'agit pour l'organisation de se présenter à son public et de décrire ses activités, le rapport annuel constitue le moyen de communication le plus stratégique. De nombreux travaux soulignent ainsi la complémentarité des sections narratives et financières des rapports annuels (Smith et Tafler, 1995), complémentarité qui fournit des informations cruciales quant à l'importance stratégique que revêtent certaines questions pour l'organisation. Le rapport annuel constitue donc un outil de communication stratégique pour l'organisation et celle-ci va ainsi chercher à se dépeindre sous des traits positifs afin de recevoir l'adhésion et de susciter l'approbation de ses parties prenantes (Elsbach, 1994). Ceci est particulièrement significatif dans le contexte de la publication d'informations relatives à l'environnement. Il n'existe en effet que très peu d'obligations en matière de communication environnementale aux États-Unis, à l'exception du TRI (Toxic Release Inventory) qui oblige depuis 1987 les entreprises appartenant à certaines industries à publier le détail de leurs émissions dans l'air, l'eau et la terre. Les organisations sont également contraintes de discuter de leur performance dans le rapport $10 \mathrm{~K}$ qu'elles remplissent pour la SEC. En dehors de ces obligations légales, toute publication d'information relative au comportement environnemental de l'organisation relève d'une décision purement stratégique pour influencer les perceptions de ses parties prenantes.

\section{Opérationnalisation des variables}

\section{Réputation}

Fortune publie chaque année les résultats d'une enquête qui évalue la réputation des 1000 plus grandes entreprises américaines. Cette enquête se fonde sur les réponses de 
top managers, directeurs et analystes financiers qui évaluent l'organisation sur la base de 8 critères ${ }^{1}$, tels que la performance financière, le degré d'innovation, ou encore la qualité des produits. Les évaluations recueillies sur ces 8 critères sont ensuite agrégées pour former un score global de réputation allant de 0 (mauvaise) à 10 (excellente). Nous avons donc utilisé ce score de réputation globale comme variable dépendante dans nos modèles, tout en étant conscients des limites de cet indicateur.

\section{Signal visible de conformité ${ }^{2}$}

Cette variable binaire mesure la présence ou l'absence d'un rapport publié par l'organisation pour rendre compte spécifiquement de sa performance environnementale. Il peut s'agir d'un rapport environnemental, d'un rapport EHetS (Environment, Health and Safety), ou d'un rapport de responsabilité sociétale abordant la dimension environnementale. Ce rapport représente un signal visible (il est indépendant du rapport annuel) de conformité (il vise à mettre en valeur l'attention que l'organisation porte à l'environnement). Nous avons codé 1 si un tel rapport existait, 0 autrement.

\section{Signal peu visible de conformité}

Cette variable binaire mesure la présence ou l'absence de mentions environnementales au sein des sections narratives du rapport annuel. Nous considérons ces mentions comme des signaux peu visibles car elles ne représentent qu'une partie du discours de l'organisation et sont entourées par des informations sans rapport avec la responsabilité environnementale de l'organisation. La force de ces signaux est donc diluée, contrairement à ceux émis dans un rapport spécifiquement dédié aux questions environnementales. Nous avons codé 1 s'il était fait mention de la performance environnementale de l'organisation, de l'impact de ses activités sur l'environnement ou encore de sa position vis-à-vis de sa responsabilité environnementale, et 0 autrement.

\section{Signal de conformité}

Cette variable, que nous utilisons exclusivement pour tester $\mathrm{H} 1$, est construite à partir de l'agrégation des deux mesures de conformité précédemment mentionnées. La variable prend la valeur 1 si l'organisation a publié un rapport environnemental indépendamment de son rapport annuel et/ou si elle fait mention de l'environnement dans les parties narratives $\mathrm{du}$ rapport annuel, 0 autrement.

\section{Signal de non-conformité}

Cette variable binaire mesure la publication d'informations négatives quant à la performance environnementale de l'organisation (e.g. amendes pour violations des réglementations en matière de protection de l'environnement) au sein de la section financière du rapport annuel ou du rapport $10 \mathrm{~K}$. Cette variable est codée 1 s'il est fait mention

\footnotetext{
1. Selon les propres termes de Fortune, ces items sont: Asset use, community and environmental friendliness, ability to develop key people, financial soundness, degree of innovativeness, investment value, management quality, and product quality.

2. Nous avons, à l'origine, construit une variable ordinale prenant en compte les différentes informations contenues dans les discours des entreprises, via l'utilisation d'une échelle développée par Wiseman (1982). Les résultats obtenus étaient similaires à ceux présentés ici. Les effets étant plus marqués en présence d'une variable dichotomique (absence vs. présence du signal) que d'une variable prenant en compte les nuances du discours, nous restreignons notre présentation aux variables dichotomiques.
} 
d'événement de non-conformité vis-à-vis des attentes et des normes en matière de performance environnementale, 0 autrement.

\section{Variables de contrôle}

Des travaux précédents suggèrent que la taille, l'âge et la performance d'une organisation sont susceptibles d'avoir un impact positif sur la réputation (Deephouse et Carter, 2005; Fombrun et Shanley, 1990). Nous avons donc inclus ces variables dans nos modèles. L'âge est opérationnalisé par le log du nombre d'années où l'organisation a fonctionné et la taille par le log des actifs totaux de l'organisation. La performance est quant à elle mesurée via son ROA. Les questions environnementales n'ayant pas la même importance dans toutes les industries ni selon les années, nous avons également inclus dans nos modèles deux variables nous permettant de contrôler par la sensibilité de l'industrie aux questions environnementales et par la variabilité des années.

Par ailleurs, pour éviter une focalisation exclusive sur les signaux émis par des sources internes à l'organisation, et parce que la couverture médiatique d'une organisation peut avoir une influence sur la manière dont elle va être perçue par ses parties prenantes (Fombrun et Shanley, 1990), nous avons ajouté dans nos modèles une variable permettant de mesurer la tonalité (négative ou positive) et la visibilité (quantité totale d'articles) de la couverture médiatique d'une organisation donnée. Conformément aux travaux qui suggèrent que les médias participent activement à la construction des réalités sociales qu'ils couvrent (Clayman et Reisner, 1998; Rindova et al., 2006), nous contrôlons le possible impact intermédiaire de la couverture médiatique sur les perceptions des par- ties prenantes. La variable Couverture médiatique mesure donc la quantité et la tonalité des informations publiées dans la presse américaine format papier et électronique sur le comportement environnemental de l'organisation. Via Lexis-Nexis, nous avons donc collecté tous les articles mentionnant le comportement environnemental des organisations appartenant à notre échantillon, sur la période 2001-2004. Cette procédure nous a permis de récolter un total de 2156 articles pertinents. Ces articles ont été codés en suivant la procédure suggérée par Deephouse et Carter (2005). Chaque article équivaut à une unité statistique et est codé positif ou négatif en fonction de la description des activités de l'organisation en matière de respect/nonrespect de l'environnement. Nous avons ensuite créé une mesure annuelle de tonalité pour chaque organisation en utilisant le coefficient Jadis-Fadner, qui nous permet de mesurer le nombre relatif de mentions positives ( $p$ ) ou négatives (n) du comportement environnemental de l'organisation sur une année donnée. La formule utilisée est la suivante:

Tonalité $=\left\{\begin{array}{l}\left(p^{2}-p \cdot n\right) /(p+n)^{2} \text { if } p>n \\ 0 \text { if } p=n ; \text { and } \\ \left(p \cdot n-n^{2}\right) /(p+n)^{2} \text { if } n>p\end{array}\right.$

Cette variable va de -1 (couverture totalement négative) à 1 (couverture totalement positive), 0 indiquant un équilibre entre les mentions positives et négatives. Nous avons ensuite calculé la variable finale en multipliant ce score de tonalité avec l'intensité de la couverture médiatique $(\log$ de $(p+n))$ pour chaque année.

Enfin, parce qu'il existe une certaine dépendance de sentier dans la construction de la 
réputation, nous avons inclus une mesure de la réputation passée de l'organisation. Pour ce faire, nous avons utilisé la variation de score de réputation entre $\mathrm{t}-2$ et $\mathrm{t}-1$, ce qui nous permet de capturer la tendance sous-jacente à l'évolution de la réputation.

\section{Modèles}

Nous disposons de données sur les 90 organisations pour les quatre années d'observation. En raison d'un décalage d'un an cependant, le nombre maximum d'observations est de 270 (90*3 années), et pour des questions d'informations manquantes dans 11 cas, le nombre total est de 259. Comme la décision de communiquer n'est pas attribuable aléatoirement aux organisations et que la communication environnementale et la réputation d'une organisation sont susceptibles de dépendre conjointement de facteurs non observables (Hamilton et Nickerson, 2003), nous avons utilisé une procédure qui permet de corriger pour l'effet d'endogénéité (Greene, 2005). Nous avons par ailleurs inclus une mesure laggée de la variation passée de la réputation afin de prendre en compte les effets de dépendance temporelle.

\section{IV - RÉSULTATS}

Le tableau 1 montre les corrélations des variables utilisées pour tester nos hypothèses. Le tableau 2 présente sept modèles. Le modèle 1 est un modèle de régression avec effets aléatoires qui inclut toutes les variables de contrôle. Les modèles 2 et 3 sont les modèles corrigeant l'endogénéité qui introduisent deux variables indépendantes et testent les hypothèses $\mathrm{H} 1 \mathrm{a}$ et $\mathrm{H} 1 \mathrm{~b}$. Le premier modèle nous permet de constater que les variables de contrôle Taille, Couverture médiatique et Performance, contri- buent significativement à améliorer la réputation. Le modèle 2 introduit la variable Signal de conformité et montre que la présence d'un tel signal a un impact positif significatif sur la réputation de l'organisation. Le modèle 3 introduit la variable Signal de non-conformité dans l'équation et montre que ce type de signal a un impact négatif significatif sur la réputation organisationnelle. Ainsi, les signaux de conformité ont un impact positif significatif sur la réputation organisationnelle tandis que les signaux de non-conformité ont un impact négatif, ce qui apporte donc un support empirique aux hypothèses H1a et H1b.

Le modèle 4 désagrège Signal de conformité en ses deux composantes - Signal de conformité visible et Signal de conformité peu visible - afin de tester l'hypothèse $\mathrm{H} 2$. Le modèle 5 introduit le terme d'interaction entre Signal de conformité visible et Signal de non-conformité afin de tester l'hypothèse $\mathrm{H} 3$.

L'hypothèse H2 suggérait qu'un signal de conformité visible aurait un impact positif plus important sur la réputation de l'organisation, qu'un signal de conformité peu visible. Cette hypothèse reçoit un support empirique dans le modèle 4. L'hypothèse H3, qui suggérait que la présence d'un signal de conformité visible devrait modérer positivement l'impact négatif d'un signal de non-conformité sur la réputation reçoit également un support empirique dans le modèle 5 puisque le coefficient du terme d'interaction est positif et significatif $(p<.05)$.

Enfin, nos dernières hypothèses émettaient l'idée que les signaux de conformité peu visibles auraient un impact différent sur la réputation en fonction de la réputation antérieure de l'organisation. Nous avons divisé 
Tableau 1 - Statistiques descriptives et corrélations

\begin{tabular}{|c|c|c|c|c|c|c|c|c|c|c|c|c|c|c|}
\hline $\mathrm{N}=259$ & Moy. & s.d. & Min & Max & Rép. & Taille & Âge & Perf. & $\begin{array}{l}\text { Rép. } \\
\text { Ant. }\end{array}$ & $\begin{array}{l}\text { Couv. } \\
\text { Méd. }\end{array}$ & S.C & SVC & SPVC & $*$ \\
\hline Réputation & 6,17 & 1,02 & 3,56 & 8,52 & & & & & & & & & & \\
\hline Taille & 8,85 & 1,41 & 6,23 & 13,92 & 0,30 & & & & & & & & & \\
\hline Âge & 3,86 & 0,94 & 0,69 & 5,15 & 0,17 & 0,06 & & & & & & & & \\
\hline Performance & 4,31 & 6,86 & $-38,12$ & 18,66 & 0,30 & 0,00 & 0,12 & & & & & & & \\
\hline $\begin{array}{l}\text { Réputation } \\
\text { antérieure }\end{array}$ & 0,08 & 0,63 & $-2,45$ & 2,11 & 0,23 & $-0,09$ & 0,08 & $-0,06$ & & & & & & \\
\hline $\begin{array}{l}\text { Couverture } \\
\text { médiatique }\end{array}$ & 0,05 & 0,84 & $-2,57$ & 3,14 & 0,10 & $-0,06$ & $-0,03$ & 0,00 & 0,06 & & & & & \\
\hline $\begin{array}{l}\text { Signal } \\
\text { de conformité }\end{array}$ & 0,27 & 0,44 & 0,00 & 1,00 & 0,21 & 0,25 & 0,09 & 0,05 & 0,04 & 0,19 & & & & \\
\hline $\begin{array}{l}\text { S. visible } \\
\text { de conformité }\end{array}$ & 0,19 & 0,40 & 0,00 & 1,00 & 0,26 & 0,28 & 0,18 & 0,03 & 0,04 & 0,25 & 0,76 & & & \\
\hline $\begin{array}{l}\text { S. peu visible } \\
\text { de conformité }\end{array}$ & 0,19 & 0,39 & 0,00 & 1,00 & 0,16 & 0,09 & 0,04 & 0,05 & 0,01 & 0,13 & 0,79 & 0,44 & & \\
\hline $\begin{array}{l}\text { Signal de } \\
\text { non-conformité }\end{array}$ & 0,60 & 0,49 & 0,00 & 1,00 & $-0,19$ & $-0,11$ & $-0,08$ & $-0,19$ & $-0,06$ & $-0,09$ & 0,07 & 0,00 & 0,17 & \\
\hline $\begin{array}{l}\text { S. de non- } \\
\text { conformité } \\
* \mathrm{~S} \text {. visible } \\
\text { de conformité }\end{array}$ & 0,12 & 0,32 & 0,00 & 1,00 & 0,19 & 0,19 & 0,06 & $-0,05$ & 0,00 & 0,16 & 0,59 & 0,74 & 0,50 & 0,24 \\
\hline
\end{tabular}


Tableau 2 - Effets sur la réputation

\begin{tabular}{|c|c|c|c|c|c|c|c|}
\hline & Modèle 1 & Modèle 2 & Modèle 3 & Modèle 4 & Modèle 5 & $\begin{array}{c}\text { Modèle } 6 \\
\text { Rep. élevée }\end{array}$ & $\begin{array}{l}\text { Modèle } 7 \\
\text { Rep. basse }\end{array}$ \\
\hline Constant & $\begin{array}{c}3.290 * * * \\
(.701)\end{array}$ & $\begin{array}{c}3.863 * * * \\
(.444)\end{array}$ & $\begin{array}{c}4.290 * * * \\
(.475)\end{array}$ & $\begin{array}{c}4.066^{* * * *} \\
(.469)\end{array}$ & $\begin{array}{c}4.151^{* * * *} \\
(.468)\end{array}$ & $\begin{array}{c}6.769 * * * \\
(.325)\end{array}$ & $\begin{array}{c}4.522 * * * \\
(.747)\end{array}$ \\
\hline Contrôle pour l'année & oui & Oui & oui & oui & oui & oui & oui \\
\hline Contrôle pour l'industrie & oui & Oui & oui & oui & oui & oui & oui \\
\hline Taille & $\begin{array}{l}.236 * * * \\
(.066)\end{array}$ & $\begin{array}{l}.180 * * * \\
(.043)\end{array}$ & $\begin{array}{l}.157 * * * \\
(.044)\end{array}$ & $\begin{array}{l}.177 * * * \\
(.043)\end{array}$ & $\begin{array}{l}.171 * * * \\
(.043)\end{array}$ & $\begin{array}{l}.112^{\dagger} \\
(.061)\end{array}$ & $\begin{array}{l}.076 \\
(.058)\end{array}$ \\
\hline Âge & $\begin{array}{l}.116 \\
(.098)\end{array}$ & $\begin{array}{c}.058 \\
(.059)\end{array}$ & $\begin{array}{c}.037 \\
(.060)\end{array}$ & $\begin{array}{c}.055 \\
(.060)\end{array}$ & $\begin{array}{c}.061 \\
(.059)\end{array}$ & $\begin{array}{l}-.044 \\
(.077)\end{array}$ & $\begin{array}{l}.018 \\
(.062)\end{array}$ \\
\hline Performance & $\begin{array}{l}.028 * * * \\
(.007)\end{array}$ & $\begin{array}{l}.044 * * * \\
(.008)\end{array}$ & $\begin{array}{l}.040 * * * \\
(.008)\end{array}$ & $\begin{array}{l}.040 * * * \\
(.008)\end{array}$ & $\begin{array}{l}.040 * * * \\
(.008)\end{array}$ & $\begin{array}{l}.028 * \\
(.012)\end{array}$ & $\begin{array}{l}.017 * \\
(.007)\end{array}$ \\
\hline Réputation antérieure & $\begin{array}{l}.446 * * * \\
(.051)\end{array}$ & $\begin{array}{l}.434 * * * \\
(.087)\end{array}$ & $\begin{array}{l}.422 * * * \\
(.086)\end{array}$ & $\begin{array}{l}.409 * * * \\
(.088)\end{array}$ & $\begin{array}{l}.411 * * * \\
(.087)\end{array}$ & $\begin{array}{c}.698 * * * \\
(.094)\end{array}$ & $\begin{array}{l}.701 * * * \\
(.089)\end{array}$ \\
\hline Signal de conformité & & $\begin{array}{l}.665 * * \\
(.230)\end{array}$ & $\begin{array}{l}.787 * * * \\
(.235)\end{array}$ & - & - & - & - \\
\hline Signal de conformité visible & & & & $\begin{array}{l}.758 * * \\
(.307)\end{array}$ & $\begin{array}{l}.538^{\dagger} \\
(.312)\end{array}$ & $\begin{array}{l}.791 * * \\
(.325)\end{array}$ & $\begin{array}{l}-.129 \\
(.596)\end{array}$ \\
\hline Signal de conformité peu visible & & & & $\begin{array}{c}.044 \\
(.165)\end{array}$ & $\begin{array}{l}-.065 \\
(.169)\end{array}$ & $\begin{array}{l}.243^{\dagger} \\
(.130)\end{array}$ & $\begin{array}{c}.088 \\
(.202)\end{array}$ \\
\hline Signal de non-conformité & & & $\begin{array}{l}-.297^{*} \\
(.120)\end{array}$ & $\begin{array}{c}-.239^{*} \\
(.118)\end{array}$ & $\begin{array}{c}-.326^{* *} \\
(.125)\end{array}$ & $\begin{array}{c}-.418^{* *} \\
(.137)\end{array}$ & $\begin{array}{c}-.236^{\dagger} \\
(.127)\end{array}$ \\
\hline $\begin{array}{l}\text { S. de non conformité } \\
* \text { S. de conformité visible }\end{array}$ & & & & & $\begin{array}{l}.561 * \\
(.266)\end{array}$ & & \\
\hline $\begin{array}{l}\text { Wald } \mathrm{Chi}^{2} \\
\mathrm{~N}\end{array}$ & $\begin{array}{c}160.8 \\
259\end{array}$ & $\begin{array}{c}117.7 \\
259\end{array}$ & $\begin{array}{c}125.0 \\
259\end{array}$ & $\begin{array}{c}119.9 \\
259\end{array}$ & $\begin{array}{c}125.1 \\
259\end{array}$ & $\begin{array}{c}140.7 \\
114\end{array}$ & $\begin{array}{c}124.8 \\
145\end{array}$ \\
\hline
\end{tabular}


l'échantillon en deux sous-groupes pour tester les dernières hypothèses, la séparation s'effectuant autour du score de réputation moyen sur l'année. Le modèle complet (modèle 4) a donc été testé indépendamment sur chaque sous-échantillon. Le modèle 6 présente les résultats pour les organisations bénéficiant d'une réputation plus élevée que la moyenne, le modèle 7 les résultats pour les organisations ayant une réputation inférieure à la moyenne de l'échantillon. L'effet de la variable Signal de conformité peu visible sur la réputation organisationnelle est significatif pour les entreprises dotées d'une réputation supérieure à la moyenne et non significatif dans l'autre cas, apportant un support empirique aux hypothèses $\mathrm{H} 4 \mathrm{a}$ et $\mathrm{H} 4 \mathrm{~b}$. Il est à noter également que l'impact de la couverture médiatique est plus favorable aux entreprises à faible réputation (modèle 7) qu'à leurs contreparties déjà reconnues.

\section{V - DISCUSSION ET CONCLUSION}

Les parties prenantes construisent leurs évaluations des organisations en fonction des informations disponibles sur leurs activités (Fombrun et Shanley, 1990), et les organisations peuvent améliorer leur réputation en envoyant des signaux positifs à propos d'elles-mêmes. Cependant, peu d'études se sont penchées sur l'impact des signaux de performance environnementale sur la réputation organisationnelle. Nous cherchons donc dans ce papier à mettre en exergue la manière dont la nature et les caractéristiques de signaux émis par les organisations peuvent affecter leur réputation.

À l'instar de recherches précédentes, nos résultats suggèrent que dans des contextes caractérisés par des asymétries d'informa- tion et une incertitude significative, le marché va utiliser des sources d'information secondaires pour remplacer des critères objectifs de qualité non observables et évaluer la crédibilité de ces signaux au travers du filtre d'un attribut organisationnel pertinent dans le contexte. La performance environnementale d'une organisation étant difficilement observable directement, les parties prenantes dépendent de la communication environnementale des organisations pour évaluer la qualité de leur management environnemental. Nos résultats suggèrent ainsi que des signaux conformes aux attentes de l'environnement institutionnel améliorent la réputation de l'organisation, tandis que des signaux non conformes la détériorent. Ils suggèrent également que ces signaux sont interprétés en fonction de leur contexte. En nous fondant sur l'idée que les évaluations vont dépendre non pas seulement de la nature du message transmis par le signal mais également de la cohérence des signaux envoyés, nous examinons les conséquences de la présence simultanée de signaux conformes et non conformes. La présence de signaux conformes et visibles semble ainsi modérer positivement la présence de signaux non conformes.

Les parties prenantes étant quasiment exclusivement dépendantes des informations communiquées par l'organisation pour évaluer sa performance, nous émettons l'hypothèse qu'elles vont tenter de réduire l'asymétrie d'information en utilisant un filtre (Weiss, 1995) destiné à évaluer la crédibilité des signaux envoyés. Comme il s'agit d'un contexte où la conformité aux normes et aux attentes sociales (Meyer et Rowan, 1977) est prédominante, nous suggérons que le filtre utilisé est d'ordre social et proposons que les parties prenantes vont utiliser la réputa- 
tion passée de l'organisation pour évaluer la crédibilité de ses signaux. Une bonne réputation antérieure offre en effet une certaine garantie que l'organisation se montrera réticente à envoyer des signaux en décalage avec la réalité, de peur de mettre en danger cette réputation déjà acquise.

Sander et Boivie (2004) montrent que les mécanismes de gouvernance peuvent être utilisés par les investisseurs pour évaluer des organisations dans des secteurs émergents tels que le e-commerce. Nous allons un cran plus loin en décrivant une situation plus encastrée où les signaux émis par les organisations sont interprétés et évalués en fonction de la crédibilité de l'organisation émettrice et où le filtre n'est utilisé que lorsque la nature du signal est sujette à caution, comme nous l'observons dans le cas de signaux de conformité peu visibles. Ce résultat converge avec des recherches antérieures adoptant une perspective institutionnelle à l'égard de la réputation et mettant en lumière l'absence de symétrie dans un champ organisationnel donné entre les acteurs bénéficiant d'une bonne réputation et les autres (Durand et al., 2007). Les organisations dotées d'une meilleure réputation recevront un meilleur crédit pour leurs actions que leurs congénères. Comme le montrent les études en sociologie économique (Phillips et Zuckerman, 2001), il existe un lien de causalité entre les évaluations précédentes, les choix stratégiques et les évaluations futures.

Ce papier apporte plusieurs contributions. En premier lieu, bien que la théorie des signaux soit largement utilisée dans la littérature économique, il n'existe à ce jour que peu d'études empiriques examinant les relations causales entre des actions symboliques (discours écrits et oraux) et des variables organisationnelles stratégiques (Suddaby et Greenwood, 2005). Plus précisément, la littérature en théorie des organisations n'a jusqu'à présent prêté que peu d'attention au processus par lequel l'utilisation d'une rhétorique de persuasion pouvait affecter directement des variables organisationnelles cruciales telles que l'accès aux ressources, la structure des marchés ou encore la performance (Suddaby et Greenwood, 2005). Cet article s'efforce donc de pallier ce manque en examinant l'impact des discours environnementaux sur une variable clé pour l'organisation, sa réputation.

Cette étude apporte une seconde contribution via l'utilisation d'une approche qui permet de différencier les impacts des signaux sur la réputation organisationnelle. En effet, au lieu de considérer les signaux comme des éléments homogènes, nous cherchons à prendre en compte leur diversité et leurs interactions afin d'évaluer plus finement leur impact sur cette variable stratégique. Suivant le travail de Heil et Robertson (1991) sur les signaux concurrentiels, qui suggère que le processus d'interprétation d'un signal dépend en partie des caractéristiques du signal (clarté, cohérence, agressivité), nous montrons non seulement que la visibilité et la cohérence des signaux émis par l'organisation a un impact sur la manière dont la réputation va être évaluée par ses parties prenantes, mais également que dans un contexte où un signal interprété négativement à été émis, la présence d'un signal positif visible peut jouer un effet tampon sur cette évaluation négative.

Troisièmement, cette étude contribue également à la littérature sur le management de la réputation, dans la mesure où nous examinons l'impact direct de la communication d'une organisation sur sa réputation, et 
non un impact indirect qui passerait par l'intervention de porte-parole. Pollock et Rindova (2003) ont montré que la présence d' " infomediaries » (intermédiaires en matière de transmission d'information) avait une influence sur la manière dont les récepteurs percevaient et évaluaient la désirabilité du comportement de l'organisation. Les médias participant activement à la construction de l'image des organisations (Rindova et al., 2006), notre contrôle de la couverture médiatique reçue par les organisations, nous permet de parvenir à une estimation plus claire de l'impact direct de la communication de l'organisation sur sa réputation.

Enfin, ces résultats contribuent à la littérature sur la communication environnementale. À l'instar de Bansal et Clelland (2004) qui montrent que la communication environnementale des organisations peut avoir un impact positif sur la variation du cours de leur action, nos résultats suggèrent clairement que la communication environnementale exerce une influence sur la manière dont l'organisation est perçue par ses diverses parties prenantes. Cette étude attire donc notre attention sur la nature et la visibilité des différents messages émis par une organisation dans un contexte caractérisé par des pressions isomorphiques importantes.

Notons pour conclure que plusieurs limites de cette étude offrent des voies de recherche futures. Tout d'abord, alors que la réputation peut être considérée comme un concept multidimensionnel (Fombrun, 1996; Rindova et al., 2005), nous ne sommes pas parvenus à séparer différentes dimensions et avons donc employé un indicateur unidimensionnel de réputation. Il serait donc intéressant d'examiner plus avant l'impact des différents signaux sur différentes dimensions de la réputation. Ensuite, l'indicateur de réputation utilisé dans cette étude - le score de Fortune - n'est pas sans failles en ce sens où il agrège plusieurs dimensions de la réputation, est fortement corrélé avec la performance financière de l'organisation, et ne permet pas de distinguer entre les représentations potentiellement différentes des parties prenantes. Ainsi serait-il intéressant d'utiliser des indicateurs différents qui permettraient de mettre en valeur la pluralité des évaluations des parties prenantes (Mitchell et al., 1997). Notre intuition est que des parties prenantes différentes pourraient évaluer différemment la réputation de l'organisation, selon la nature et les caractéristiques du signal. Enfin, il s'agirait de s'intéresser aux impacts différenciés de la couverture médiatique sur la réputation des organisations. Nos résultats suggèrent en effet qu'il existe un effet de seuil après lequel une couverture positive semble être plus profitable aux organisations ayant une réputation inférieure à la moyenne.

\section{Bibliographie}

Bansal P., "Evolving sustainability: A longitudinal study of organizational sustainable development", Strategic Management Journal, vol. 26, n 3, March 2005, p. 197-218.

Bansal P., Clelland I., "Talking trash: Legitimacy, impression management, and unsystematic risk in the context of the natural environment", Academy of Management Journal, vol. 47, n 1, 2004, p. 93-103. 
Baron D., "Private politics, corporate social responsibility and integrated strategy", Journal of Economics and Management Strategy, 10, 2001, p. 7-45.

Benjamin B. et Podolny J.M., "Status, quality, social order in the California wine industry, Administrative Science Quarterly, vol. 44, 1999, p. 563-589.

Clayman S.E. et Reisner A., "Gatekeeping in action: Editorial conferences and assessments of news-worthiness", American Sociological Review, vol. 63, 1998, p. 178-199.

Crane A., "Corporate greening as amoralization", Organization Studies, 21, 2000, p. 673-696.

Deephouse D.L., "Media reputation as a strategic resource: An integration of mass communication and resource-based theories", Journal of Management, vol. 26, $\mathrm{n}^{\circ}$ 6, 2000, p. 1091-1112.

Deephouse D.L. et Carter S.M., "An examination of differences between organizational legitimacy and organizational reputation", Journal of Management Studies, vol. 42, n 2, 2005, p. 329-360.

DiMaggio P.J. et Powell W.W., "The iron cage revisited: Institutionalized isomorphism and collective rationality in organization fields", American Sociological Review, vol. 48, $\mathrm{n}^{\circ} 2$, 1983, p. 147-160.

Durand R., Rao H. et Monin P., "Code and conduct in French cuisine: Impact of codechanges on external evaluations", Strategic Management Journal, vol. 28, $\mathrm{n}^{\circ}$ 5, 2007, p. 455-472.

Elsbach K.D., "Managing organizational legitimacy in the California cattle industry: The construction and effectiveness of verbal accounts", Administrative Science Quarterly, vol. 39, 1994, p. 57-88.

Elsbach K.D. et Sutton R.I., “Acquiring organizational legitimacy through illegitimate actions: a marriage of institutional and impression management theories", Academy of Management Journal, vol. 35, $\mathrm{n}^{\circ}$ 4, 1992, p. 699-738.

Fombrun C.J., Reputation: Realizing value from the corporate image, Harvard Business School Press, Boston, MA, 1996.

Fombrun C.J. et Shanley M., "What's in a name? Reputation building and corporate strategy", Academy of Management Journal, 33, 1990, p. 233-258.

Gardberg N.A. et Fombrun C.J., "Corporate citizenship : Creating intangible assets across institutional environments", Academy of Management Review, vol. 31, $\mathrm{n}^{\circ}$ 2, 2006, p. 329-346.

Glynn M.A. et Abzug R., "Institutionalizing identity: Symbolic isomorphism and organizational names", Academy of Management Journal, vol. 45, n 1, 2002, p. 267-280.

Greene W.H., Econometric analysis, Prentice Hall, Upper Saddle River, 2003.

Hamilton B.H., Nickerson J.A., "Correcting for endogeneity in strategic management research", Strategic Organization, vol.1, n 1, 2003, p. 51-78.

Heil O. et Robertson T.S., "Toward a theory of competitive market signalling: A research agenda", Strategic Management Journal, vol. 12, n 6, September 1991, p. 403-418. 
King A.A. et Lenox M.J., "The strategic use of decentralized institutions : Exploring certification with the ISO 14001 management standard", Academy of Management Journal, vol. 48, n 6, 2005, p. 1091-1106.

Kreps D.M. et Wilson R., "Reputation and imperfect information", Journal of Economic Theory, 27, 1982, p. 253-279.

Martinet A.C. et Reynaud E., "Shareholders, stakeholders et stratégie », Revue française de gestion, $\mathrm{n}^{\circ} 136$, novembre-décembre 2001, p. 12-25.

McGuire J.B., Sundgren A., Schneeweis T., "Corporate social responsibility and firm financial performance”, Academy of Management Journal, 31, 1988, p. 854-872.

Meyer J.W. et Rowan B., "Institutionalized organizations : Formal structure as myth and ceremony”, American Journal of Sociology, vol. 83, n², 1977, p. 340-363.

Milgrom P. et Roberts J., "Predation, reputation, and entry deterrence", Journal of Economic Theory, 27, 1982, p. 280-312.

Mitchell R.K., Agle B.R., Wood D.J., "Toward a theory of stakeholder identification and salience: Defining the Principle of Who or What Really Counts", Academy of Management Review, vol. 22, n 4, 1997, p. 853-886.

Oliver C., "Strategic responses to institutional processes", Academy of Management Review, vol. $15, \mathrm{n}^{\circ} 1,1991, \mathrm{p} .145-17$.

Phillips D. et Zuckerman E., "Middle-status conformity: Theoretical restatement and empirical demonstration in two markets", American Journal of Sociology, 107, 2001, p. 379-429.

Pollock T.G. et Rindova V.P., "Media legitimation effects in the market for initial public offerings", Academy of Management Journal, vol. 46, n 5, 2003, p. 631-642.

Rindova V.P., Pollock T.G. et Hayward M.A., "Celebrity firms: The social construction of market popularity", Academy of Management Review, vol. 31, n 1, 2006, p. 50-71.

Rindova V.P., Williamson I.O., Petkova A.P. et Sever J.M., "Being good or being known: An empirical examination of the dimensions, antecedents, and consequences of organizational reputation", Academy of Management Journal, vol. 48, n 6, 2005, p. 1033-1049.

Roberts P.W. et Dowling G.R., "Corporate reputation and sustained superior financial performance", Strategic Management Journal, vol. 23, n 12, December 2002, p. 1077-1093.

Smith M. et Tafler R., "The incremental effect of narrative accounting information in organizational annual reports", Journal of Business Finance and Accounting, vol. 22, n 8, 1995, p. 1195-1210.

Spence A.M., Market signaling: Information transfer in hiring and related processes. Harvard University Press, Cambridge, MA, 1974.

Suchman M.C., "Managing legitimacy: Strategic and institutional approaches", Academy of Management Review, vol. 20, n 3, 1995, p. 571-610.

Suddaby R. et Greenwood R., "Rhetorical strategies of legitimacy", Administrative Science Quarterly, 50, 2005, p. 35-67.

Waddock S. et Graves S., "The corporate social performance - financial performance link", Strategic Management Journal, vol. 18, n 4, April 1997, p. 303-319. 
Weigelt K. et Camerer C., "Reputation and corporate strategy: A review of recent theory and applications", Strategic Management Journal, vol. 9, n ${ }^{\circ}$, September-October 1988, p. 443-454.

Weiss A., "Human capital vs. signalling: Explanation of wages", Journal of Economic Perspectives, vol. 9, n 4 , 1995, p. 133-154.

Westphal J.D. et Zajac E.J., "The symbolic management of stockholders: Corporate governance reforms and shareholder reactions", Administrative Science Quarterly, 43, 1998, p. 127-153.

Wilmshurst T.D. et Frost G.R., "Organizational environmental reporting: A test of legitimacy theory", Accounting, Auditing et Accountability Journal, vol. 13, n 1, 2000, p. 10-26.

Wiseman J., "An evaluation of environmental disclosures made in organizational annual reports", Accounting, Organizations and Society, vol. 7, n 1, 1982, p. 53-63. 
